\title{
circ-ACACA promotes proliferation, invasion, migration and glycolysis of cervical cancer cells by targeting the miR-582-5p/ERO1A signaling axis
}

\author{
DANDAN HUANG ${ }^{1}$ and CUIMEI LI ${ }^{2}$ \\ ${ }^{1}$ Department of Gynecology and Obstetrics, Inner Mongolia Baogang Hospital (The Third Affiliated \\ Hospital of Inner Mongolia Medical University), Baotou, Inner Mongolia Autonomous Region 014010; \\ ${ }^{2}$ Department of Gynecology and Obstetrics, Xi'An Fifth Hospital, Xi'An, Shaanxi 710000, P.R. China \\ Received February 23, 2021; Accepted May 6, 2021
}

DOI: $10.3892 / \mathrm{ol} .2021 .13056$

\begin{abstract}
Circular RNAs (circ) have been reported to serve crucial roles in the regulation of cancer occurrence and development. The present study aimed to investigate the role of circ-acetyl-CoA carboxylase $\alpha$ (ACACA) in the progression of cervical cancer (CC). The expression levels of circ-ACACA in several CC cell lines were first determined using reverse transcription-quantitative PCR. circ-ACACA expression was subsequently knocked down to evaluate its effects on the viability, proliferation, apoptosis, invasion and migration of CC cells using MTT, colony formation, TUNEL, transwell and wound healing assays, respectively. ${ }^{13} \mathrm{C}$-labeling of intracellular metabolites and analysis of glucose consumption and lactate production were performed to determine the levels of glycolysis. In addition, the expression levels of endoplasmic reticulum oxidoreductase $1 \alpha$ (ERO1 $\alpha$; ERO1A) and glycolysis-related proteins were analyzed using western blotting. The binding interactions among circ-ACACA, microRNA (miR)-582-5p and ERO1A were validated using dual-luciferase reporter assays. Subsequently, rescue experiments were performed to determine the potential underlying mechanism by which circ-ACACA affected CC cell functions. The results revealed that circ-ACACA expression was significantly upregulated in CC cells and silencing of circ-ACACA significantly reduced the proliferation, invasion and migration, and promoted the apoptosis of CC cells. Knockdown of circ-ACACA markedly inhibited glycolysis in CC cells. However, the effects of silencing of circ-ACACA on CC cells were reversed following transfection with the miR-582-5p
\end{abstract}

Correspondence to: Dr Cuimei Li, Department of Gynecology and Obstetrics, Xi'An Fifth Hospital, 112 Xiguanzheng Street, Lianhu, Xi'An, Shaanxi 710000, P.R. China

E-mail: licuimei66@163.com

Key words: cervical cancer, circular RNA acetyl-CoA carboxylase $\alpha$, glycolysis, apoptosis, endoplasmic reticulum disulphide oxidase $1 \alpha$ inhibitor or pcDNA3.1-ERO1A overexpression plasmid. In conclusion, to the best of our knowledge, the present study was the first to investigate the role of circ-ACACA in CC progression. The results suggested that circ-ACACA may promote $\mathrm{CC}$ tumorigenesis and glycolysis by targeting the miR-582-5p/ERO1A signaling axis. Therefore, circ-ACACA may be a promising biomarker for $\mathrm{CC}$ diagnosis and treatment.

\section{Introduction}

Cervical cancer (CC) is the second most common type of cancer in women aged 20-39 years worldwide, and represents a serious threat to public health (1). Each year, 265,700 individuals die from the disease globally, and thus, efforts have been made to improve the timely diagnosis and effective treatment of CC $(2,3)$. Currently, hysterectomy has become increasingly popular and is widely used as a treatment for early-stage CC (4). Targeted therapies have also become an effective strategy for CC treatment (5).

Circular RNAs (circRNAs/circ), a novel class of non-coding RNAs (ncRNAs), are characterized by a covalently closed loop without a 5' cap and 3' polyadenylated tail, and are generated through abnormal transcription and splicing (6). Accumulating evidence has demonstrated that circRNAs exert effects on multiple biological and pathological processes, suggesting that they may be involved in the occurrence and development of numerous diseases, including cancer $(7,8)$. For example, circ-acetyl-CoA carboxylase $\alpha$ (ACACA) has been previously reported to promote the proliferation of non-small cell lung cancer (NSCLC) cells by modulating proliferation, migration and glycolysis via microRNA (miRNA/miR)-1183 and the PI3K/AKT signaling pathway (9). However, to the best of our knowledge, the role of circ-ACACA in $\mathrm{CC}$ remains to be determined.

miRNAs are small ncRNA molecules that can sponge target genes and regulate their expression (3). A previous study demonstrated that miR-582-5p inhibits the proliferation and invasion of NSCLC cells by downregulating Notch1 expression (10). In addition, bone metastasis of prostate cancer is inhibited by the combined effects of miR-582-3p and miR-582-5p via modulation of TGF- $\beta$ signaling 
transduction (11). Long ncRNA urothelial cancer associated 1 targets miR-582-5p and promotes the progression and drug resistance of bladder cancer cells through the inhibition of autophagy related 7-mediated autophagy (12). The expression levels of miR-582-5p have also been reported to be downregulated in endometrial and gastric cancer, and miR-582-5p overexpression inhibits cell proliferation and promotes apoptosis by targeting AKT3 (13). Furthermore, the inhibitory effect of miR-582-5p on the proliferation of colorectal cancer and hepatocellular carcinoma cells has been identified to be achieved by targeting certain genes (14). However, to the best of our knowledge, the role of miR-582-5p in CC has not yet been reported.

Endoplasmic reticulum oxidoreductase $1 \alpha$ (ERO1 $\alpha$; ERO1A) is an oxidase located in the endoplasmic reticulum, which promotes the formation of disulphide bonds in granulocyte-colony stimulating factor (15). Accumulating evidence has demonstrated the close association between upregulated expression levels of ERO1A and poor prognosis in multiple types of cancer, such as pancreatic cancer, cholangiocarcinoma and breast cancer (16-19). In a previous study, knockdown of ERO1A expression reduced the proliferation, migration and tumorigenesis of $\mathrm{CC}$ cells by downregulating $\mathrm{H}_{2} \mathrm{O}_{2}$-associated epithelial-to-mesenchymal transition (20). Additionally, ERO1A has been demonstrated to affect the functions of pancreatic cancer cells by activating the Wnt/ $\beta$-catenin signaling pathway to enhance the progression of pancreatic cancer (17). A previous study also demonstrated that upregulated expression levels of ERO1A are associated with the poor prognosis of gastric cancer (21). Furthermore, endoplasmic reticulum stress-dependent ERO1A expression has been demonstrated to enhance aerobic glycolysis in pancreatic cancer (16). Hypoxia-inducible ERO1A also promotes the development of colorectal cancer by regulating integrin- $\beta 1$ and integrin- $\beta 1$-associated signaling in colorectal cancer cells (22).

Therefore, the present study aimed to investigate the potential role of circ-ACACA in CC and its regulatory effects on the miR-582-5p/ERO1A signaling axis.

\section{Materials and methods}

Cell lines and culture. Cervical squamous cell carcinoma cell lines (Ca Ski and SiHa), a endocervical adenocarcinoma cell line (HeLa) and the normal cervical epithelial cell line (End1/E6E7) were obtained from The Cell Bank of Type Culture Collection of The Chinese Academy of Sciences. All cells were cultured in DMEM (Gibco; Thermo Fisher Scientific, Inc.) supplemented with 10\% FBS (Gibco; Thermo Fisher Scientific, Inc.), $100 \mathrm{mg} / \mathrm{l}$ and streptomycin (Gibco; Thermo Fisher Scientific, Inc.) and $100 \mathrm{U} / 1$ penicillin (Gibco; Thermo Fisher Scientific, Inc.) and maintained in an atmosphere of $37^{\circ} \mathrm{C}$ with $5 \% \mathrm{CO}_{2}$.

Cell transfection. pIRES vectors containing short hairpin RNA (shRNA)-circ-ACACA\#1, shRNA-circ-ACACA\#2, shRNA-negative control (NC), miR-582-5p mimic (50 nM; sense, 5'-UUACAGUUCAACCAGUUACU-3' and antisense, 5'-UAACUGGUUGAACAACUGUAAUU-3'), mimic-NC (50 nM; sense, 5'-UUCUCCGAACGUGUCACG
UTT-3' and antisense, 5'-ACGUGACACGUUCGGAGA ATT-3'), miR-582-5p inhibitor (50 nM; 5'-AGUAACUGG UUGAACAACUGUAA-3'), NC inhibitor (50 nM; 5'-CAG UACUUUUGUGUAGUACAAA-3'), pcDNA3.1-ERO1A and pcDNA3.1-NC (empty plasmid) were purchased from Shanghai GenePharma Co., Ltd. HeLa cells $\left(1 \times 10^{6}\right.$ cells per well) were plated and incubated in six-well plates for $24 \mathrm{~h}$ and then transfected for $48 \mathrm{~h}$ with the corresponding plasmids, oligonucleotides or NCs using Lipofectamine ${ }^{\circledR} 2000$ reagent (Invitrogen; Thermo Fisher Scientific, Inc.) at $37^{\circ} \mathrm{C}$. The transfected cells were used for subsequent experiments at $48 \mathrm{~h}$ after transfection. Untreated cells were used as the control group.

Reverse transcription-quantitative PCR (RT-qPCR). Total RNA was extracted from CC cells using TRIzol ${ }^{\circledR}$ reagent (Invitrogen; Thermo Fisher Scientific, Inc.) according to the manufacturer's protocols. Total RNA was reverse transcribed into cDNA using the PrimeScript ${ }^{\mathrm{TM}}$ Strand cDNA synthesis kit (Takara Biotechnology Co., Ltd.). The temperature protocol for this step was as follows: $70^{\circ} \mathrm{C}$ for $5 \mathrm{~min}, 37^{\circ} \mathrm{C}$ for $5 \mathrm{~min}$ and $42^{\circ} \mathrm{C}$ for $1 \mathrm{~h}$. qPCR was subsequently performed using a Power SYBR ${ }^{\circledR}$ Green PCR Master mix (Invitrogen; Thermo Fisher Scientific, Inc.) on an ABI 7500 Real-Time PCR Detection system (Applied Biosystems; Thermo Fisher Scientific, Inc.). The following thermocycling conditions were used: Initial denaturation at $95^{\circ} \mathrm{C}$ for $10 \mathrm{~min}$; followed by 40 cycles of denaturation at $95^{\circ} \mathrm{C}$ for $15 \mathrm{sec}$ and annealing at $60^{\circ} \mathrm{C}$ for $1 \mathrm{~min}$; and a final extension of $10 \mathrm{~min}$ at $72^{\circ} \mathrm{C}$. Primers pairs used in this study were as follows: circ-ACACA forward, 5'-GTGGCT TTGAAGGAGCTGTC-3' and reverse, 5'-CAGACATGCTGGACCTTGAA-3'; miR-582-5p forward, 5'-GCGGTTACAGTTGTTCAACC-3' and reverse, 5'-CTCAACTGGTGTCGTGGA-3'; ERO1A forward, 5'-ATGACATCAGCCAGTGTGGA-3' and reverse, 5'-CAT GCTTGGTCCACTGAAGA-3'; GAPDH forward, 5'-ACA ACTTTGGTATCGTGGAAGG-3' and reverse, 5'-GCCATC ACGCCACAGTTTC-3'; and U6 forward, 5'-GGAACGATA CAGAGAAGATTAGC-3' and reverse, 5'-TGGAACGCT TCACGAATTTGCG-3'. The miRNA expression level was normalized to U6 and GAPDH was applied as the internal control of mRNAs, following quantification using the $2^{-\Delta \Delta C q}$ method (23).

RNase $R$ treatment assay. Six units of RNase R (Geneseed Biotech, Inc.) were added into every $2 \mu \mathrm{g}$ RNA and incubated at $37^{\circ} \mathrm{C}$ for $20 \mathrm{~min}$. After RNase R treatment, the mRNA expression of circ-ACACA and ACACA was detected by RT-qPCR.

Cell cytoplasm/nucleus fraction isolation. Cell cytoplasm/nucleus fraction isolation was performed using the Nuclear/Cytosol Fractionation Kit (Cell Biolabs, Inc.). Briefly, extracted RNAs from the cytoplasm or nucleus were determined by RT-qPCR which was performed in the previous RT-qPCR section. The relative expression levels of circ-ACACA, ACACA, nuclear control transcript (U6) and cytoplasmic control transcript ( $\beta$-actin) were measured. $\beta$-actin and U6 were used as the internal control for cytosolic and nuclear fractions, respectively. 
MTT assay. The viability of transfected HeLa cells was detected using MTT reagent (Sigma-Aldrich; Merck KGaA). Briefly, cells $\left(6 \times 10^{3}\right.$ cells/well) were plated into 96 -well plates and incubated for 24,48 or $72 \mathrm{~h}$. At each time point, $10 \mu \mathrm{l}$ MTT was added to each well. Following incubation, $100 \mu \mathrm{l}$ DMSO (Sigma-Aldrich; Merck KGaA) was added to each well to dissolve the purple formazan crystals. The absorbance of each well was measured at a wavelength of $490 \mathrm{~nm}$ using a microplate reader (Bio-Rad Laboratories, Inc.).

Colony formation assay. A total of $2 \times 10^{3} \mathrm{HeLa}$ cells/well were seeded into a six-well plate and maintained in a humidified incubator with $5 \% \mathrm{CO}_{2}$ at $37^{\circ} \mathrm{C}$. Following $48 \mathrm{~h}$ of incubation, the plates were washed with PBS twice, and then colonies were fixed with $10 \%$ paraformaldehyde for $10 \mathrm{~min}$ at room temperature and stained with $0.1 \%$ crystal violet for $15 \mathrm{~min}$ at room temperature. After the plates were washed with phosphate buffer saline (PBS) at room temperature for $1 \mathrm{~min}$ and dried, the number of colonies was counted under an inverted light microscope.

Cell invasion assay. To detect the ability of cell invasion, Transwell plates with a $8-\mu \mathrm{m}$ pore insert precoated with Matrigel (BD Biosciences) overnight at $37^{\circ} \mathrm{C}$ were obtained. A total of $2 \times 10^{4}$ transfected HeLa cells were trypsinized and plated into the upper chamber of transwell plates with serum-free DMEM. The lower chambers were filled with $800 \mu \mathrm{l}$ DMEM supplemented with $10 \%$ FBS. Following $48 \mathrm{~h}$ of incubation at $37^{\circ} \mathrm{C}$, the invasive cells were fixed with $95 \%$ ethanol for $20 \mathrm{~min}$ at $37^{\circ} \mathrm{C}$ and stained with $0.1 \%$ crystal violet for $10 \mathrm{~min}$ at $37^{\circ} \mathrm{C}$. The number of cells in five randomly selected fields of view was counted under a light microscope.

Wound healing assay. HeLa cells were plated into 96-well plates at a density of $4 \times 10^{3}$ cells/well and cultured until they reached $80 \%$ confluence. The cells were washed with PBS three times and then cultured in medium supplemented with $1 \%$ FBS for $24 \mathrm{~h}$. An artificial wound was created across the cell monolayer by scratching the center of all wells with a $10-\mu 1$ pipette tip. Migratory cells were visualized using an inverted light microscope. ImageJ software (version 1.52r; National Institutes of Health) was used to determine the wound healing rate.

TUNEL assay. Following culture for $24 \mathrm{~h}, 1 \times 10^{5} \mathrm{HeLa}$ cells were seeded in a 6 -well plate. Cells were fixed with $4 \%$ paraformaldehyde at $4^{\circ} \mathrm{C}$ for $20 \mathrm{~min}$, permeabilized with $0.1 \%$ Triton X-100 in PBS and subsequently incubated with TUNEL reagents (EMD Millipore). Cell nuclei was stained with diaminobenzene for $10 \mathrm{~min}$ at room temperature. The apoptosis of TUNEL-positive cells was observed under a fluorescence microscope (magnification, x200; Olympus Corporation) and cells were counted in five randomly selected microscopic fields.

Glycolysis assay. Glycolysis was determined using a Seahorse XF Glycolytic Rate assay kit (Agilent Technologies, Inc.) on a Seahorse XFe96 analyzer (Agilent Technologies, Inc.) according to the manufacturer's protocol. Briefly, $2 \times 10^{4}$ HeLa cells per well transfected with shRNA-circ-ACACA,
miR-582-5p, shRNA-circ-ACACA + miR-582-5p inhibitor or respective NCs were plated into a 96-well plate. After the probes were calibrated, $10 \mathrm{mmol}$ glucose, $10 \mu \mathrm{mol}$ oligomycin and $50 \mathrm{mmol} 2$-deoxyglucose were serially injected to detect the extracellular acidification rate (ECAR). Data were analyzed using Seahorse XFe24 Wave 2.2 software (Agilent Technologies, Inc.).

Glucose consumption and lactate production assays. HeLa cells and cell culture medium were collected following transfection for $48 \mathrm{~h}$. The concentration of glucose in the cell culture medium was determined using a Glucose assay kit (Sigma-Aldrich; Merck KGaA), whereas the lactate concentration was measured using a Lactic Acid assay kit (Seebio), according to the manufacturer's protocols.

Measurement of intracellular metabolite generation. ${ }^{13} \mathrm{C}$-labeled intracellular metabolites were identified as previously described (24). Briefly, $1 \times 10^{7} \mathrm{HeLa}$ cells were incubated with $2 \mathrm{~g} / 1^{13} \mathrm{C}$-labeled glucose for $2 \mathrm{~h}$ at $37^{\circ} \mathrm{C}$. Metabolites were subsequently extracted and evaluated using a liquid chromatography system equipped with a TripleTOF ${ }^{\circledR} 5600$ mass spectrometer (SCIEX; AB SCIEX). Electrospray positive ion mode was used along with the following parameters: ion voltage, 5,500 V; declustering potential, $80 \mathrm{~V}$; source temperature, $60^{\circ} \mathrm{C}$; curtain gas, $35 \mathrm{psi}$; and $100-1,000 \mathrm{~m} / \mathrm{z}$.

Western blotting. Total protein was extracted from cells using RIPA lysis buffer (Beyotime Institute of Biotechnology). Protein concentration was determined using a BCA kit (Beyotime Institute of Biotechnology) and protein samples (40 $\mu \mathrm{g}$ protein/lane) were separated via $12 \%$ SDS-PAGE. The separated proteins were subsequently transferred onto PVDF membranes (EMD Millipore) and the membranes were blocked with $5 \%$ skimmed milk for $1.5 \mathrm{~h}$ at room temperature. The membranes were then incubated with primary antibodies overnight at $4{ }^{\circ} \mathrm{C}$. Following the primary antibody incubation, the membranes were rinsed with TBS-0.2\% Tween 20 and incubated with the goat anti-rabbit horseradish peroxidase-conjugated secondary antibody (dilution, 1:2,000; cat. no. ab205718; Abcam) at room temperature for $1 \mathrm{~h}$. GAPDH was used as the internal loading control. Protein bands were visualized using an ECL detection system (Thermo Fisher Scientific, Inc.) and densitometric analysis was performed using Image J software (version 1.52r; National Institutes of Health). The following primary antibodies were used: anti-hypoxia-inducible factor $1 \alpha$ (HIF-1A; cat. no. 36169T; 1:1,000; Cell Signaling Technology, Inc.), anti-lactate dehydrogenase A (LDHA; cat. no. 3582T; 1:1,000; Cell Signaling Technology, Inc.), anti-glucose transporter type 1 (GLUT1; cat. no. 12939S; 1:1,000; Cell Signaling Technology, Inc.), anti-hexokinase-2 (HK2; cat. no. 2867S; 1:1,000; Cell Signaling Technology, Inc.), anti-ERO1A (cat. no. 3264T; 1:1,000; Cell Signaling Technology, Inc.) and anti-GAPDH (cat. no. 5174T; 1:1,000; Cell Signaling Technology, Inc.). GAPDH was used as an internal control.

Dual-luciferase reporter assay. The binding site between circ-ACACA and miR-582-5p as well as miR-582-5p and ERO1A was predicted using the Encyclopedia of RNA Interactomes 

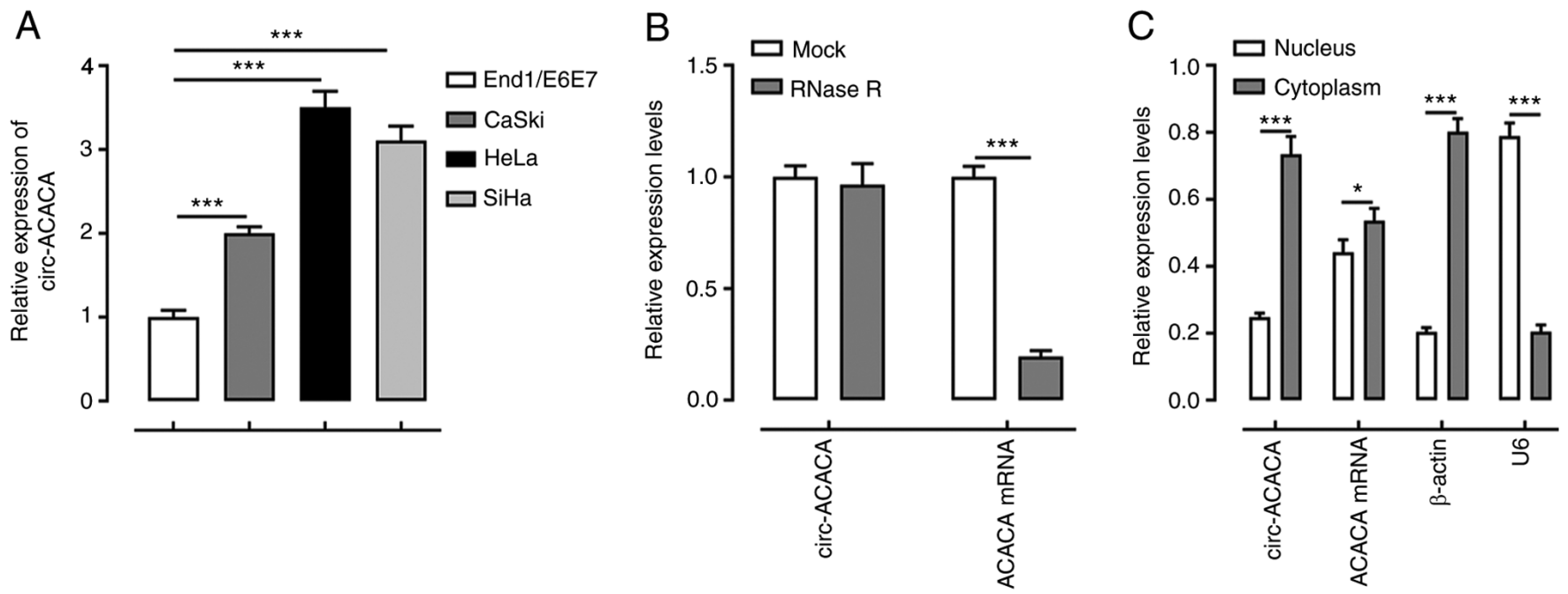

Figure 1. circ-ACACA expression is upregulated in CC cells. (A) Expression levels of circ-ACACA in various CC cell lines were determined using RT-qPCR. (B) mRNA expression levels of circ-ACACA and ACACA were analyzed using RT-qPCR. (C) mRNA expression levels of circ-ACACA, ACACA, $\beta$-actin and U6 in the nucleus and cytoplasm were determined using RT-qPCR. ${ }^{*}<<0.05,{ }^{* * * *} \mathrm{P}<0.001$. ACACA, acetyl-CoA-carboxylase $\alpha$; CC, cervical cancer; circ-ACACA, circular RNA acetyl-CoA-carboxylase $\alpha$; RT-qPCR, reverse transcription-quantitative PCR.

(ENCORI) database (http://starbase.sysu.edu.cn/). To validate the binding relationship between miR-582-5p and circ-ACACA or ERO1A, wild-type (WT) or mutant (MUT) 3'-untranslated region sequences of circ-ACACA or ERO1A were cloned into a pmiRGLO dual-luciferase miRNA target expression reporter (Promega Corporation). HeLa cells were subsequently transfected with miR-582-5p mimic or mimic-NC and WT or MUT reporters using Lipofectamine ${ }^{\circledR} 2000$ reagent (Invitrogen; Thermo Fisher Scientific, Inc.). The relative luciferase activity was measured at $48 \mathrm{~h}$ post-transfection using a Dual Luciferase Reporter assay system (Promega Corporation). And firefly luciferase activity was normalized to Renilla luciferase activity.

Statistical analysis. All experiments were repeated independently in triplicate. Statistical analysis was performed using SPSS v20.0 software (IBM Corp.). Data are presented as the mean \pm SD. Statistical differences between two groups were determined using an unpaired Student's t-test, while one-way ANOVA followed by Tukey's post hoc test was used for comparisons among $\geq 3$ groups. $\mathrm{P}<0.05$ was considered to indicate a statistically significant difference.

\section{Results}

circ-ACACA expression is upregulated in CC cells. To investigate the role of circ-ACACA in CC, its expression levels in CC cells were first determined using RT-qPCR. The results revealed that the mRNA expression levels of circ-ACACA were upregulated in CC cells compared with End1/E6E7 cells (Fig. 1A). The expression levels of circ-ACACA were the highest in HeLa cells among the cell lines examined. Therefore, HeLa cells were used as the CC cell model in subsequent experiments. As shown in Fig. 1B, circ-ACACA was not dissolved, while ACACA mRNA was dissolved by RNase R. Furthermore, circ-ACACA was expressed at a higher level in the cytoplasm compared with the nucleus (Fig. 1C).
These results suggested that circ-ACACA expression may be upregulated in CC cells.

Silencing of circ-ACACA inhibits the proliferation, invasion and migration, while promoting the apoptosis of CC cells. circ-ACACA expression was subsequently knocked down to determine its effects in CC cells. The expression levels of circ-ACACA were downregulated following transfection with shRNA-circ-ACACA\#1 and shRNA-circ-ACACA\#2 compared with shRNA-NC, especially in shRNA-circ-ACACA\#2. Therefore, shRNA-circ-ACACA\#2 was selected for use in subsequent experiments (Fig. 2A). The effects of transfection of shRNA-circ-ACACA on CC cells were determined using functional assays. MTT and colony formation assays demonstrated that cell viability and proliferation were decreased in HeLa cells transfected with shRNA-circ-ACACA as compared with the shRNA-NC group (Fig. 2B and C). Furthermore, transfection with shRNA-circ-ACACA increased the levels of apoptosis compared with the shRNA-NC group (Fig. 2D and E). circ-ACACA silencing also notably inhibited cell invasion and migration compared with the shRNA-NC group (Fig. 2F-I). These results suggested that silencing of circ-ACACA may inhibit the proliferation, invasion and migration, and promote the apoptosis of CC cells.

Silencing of circ-ACACA suppresses glycolysis in CC cells. It was subsequently investigated whether knockdown of circ-ACACA led to the attenuation of glycolysis. As displayed in Fig. 3A, ECAR was notably decreased after transfection with shRNA-circ-ACACA when compared with the shRNA-NC group. Lactate production and glucose consumption assays were performed to evaluate the effect of circ-ACACA on glycolysis. As shown in Fig. 3B and C, the transfection with shRNA-circ-ACACA markedly decreased the production of lactate and increased the levels of glucose compared with the shRNA-NC group. In addition, HeLa cells were incubated with ${ }^{13} \mathrm{C}$-labeled glucose and liquid chromatography-mass 
A

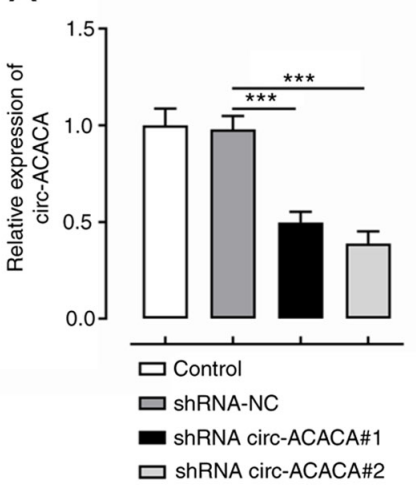

B

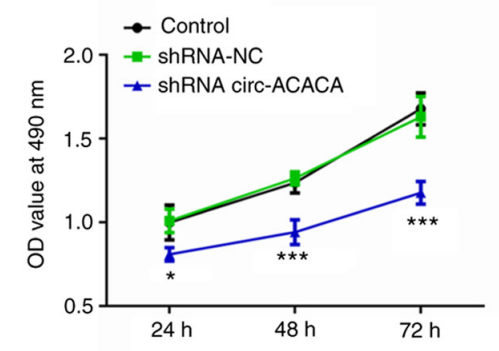

D
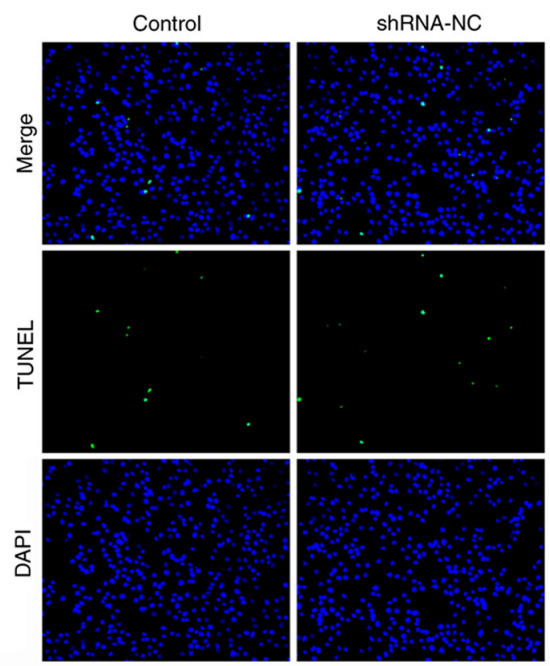

F Invasion

Control

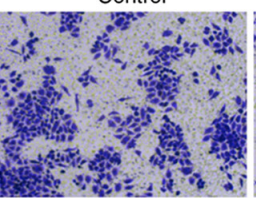

ShRNA-NC

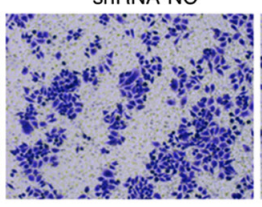

ShRNA-NC

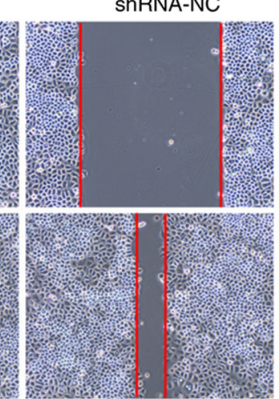

shRNA circ-ACACA
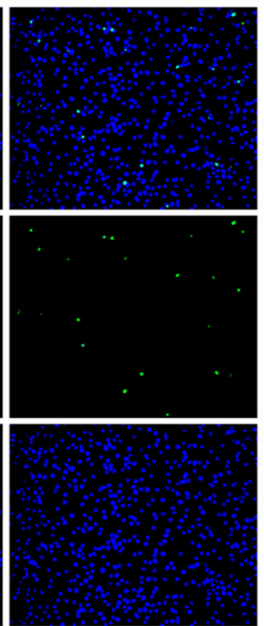

shRNA circ-ACACA
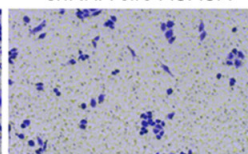

ShRNA CITC-ACACA

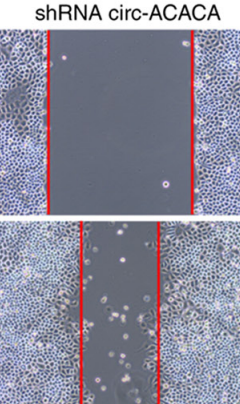

C

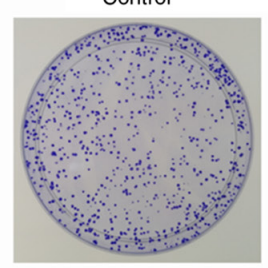

shRNA circ-ACACA

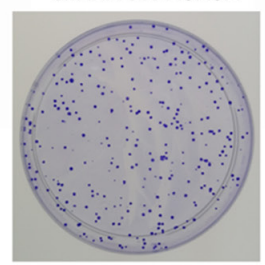

E

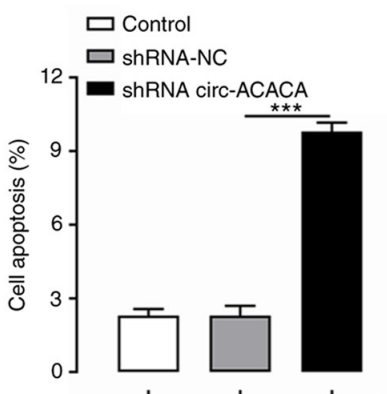

G

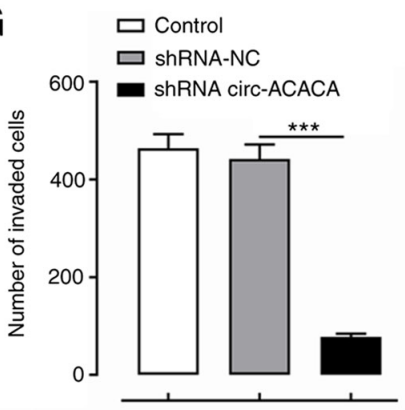

I

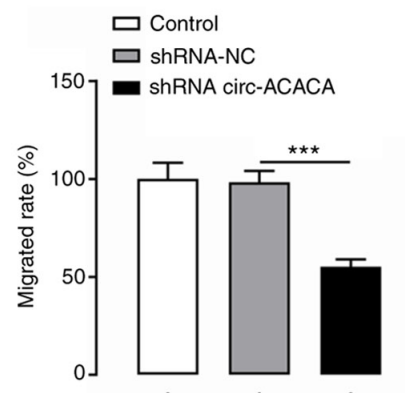

Figure 2. Knockdown of circ-ACACA inhibits the proliferation, invasion and migration, and promotes the apoptosis of cervical cancer cells. (A) Expression levels of circ-ACACA following the transfection of cells with shRNA-circ-ACACA were detected using reverse transcription-quantitative PCR. (B) Cell viability was analyzed using an MTT assay. ${ }^{*} \mathrm{P}<0.05,{ }^{* * *} \mathrm{P}<0.001$ vs. shRNA-NC. (C) Cell proliferation was determined using a colony formation assay. (D) Cell apoptosis was analyzed using a TUNEL assay. Magnification, x200. (E) The apoptosis rate of TUNEL assay. (F) HeLa cell invasion was determined using transwell assay following transfection with shRNA-circ-ACACA. Magnification, x100. (G) The number of invaded cells. (H) HeLa cell migration was tested using wound healing assay following transfection with shRNA-circ-ACACA. Magnification, $\mathrm{x} 200$. (I) The cell migration rate. ${ }^{*} \mathrm{P}<0.05,{ }^{* * * *} \mathrm{P}<0.001$. circ-ACACA, circular RNA acetyl-CoA-carboxylase $\alpha$; NC, negative control; OD, optical density; shRNA, short hairpin RNA.

spectrometry was performed to gain more in-depth insights into the metabolic flux of glucose. As shown in Fig. 3D, the ratio of 13C-marked glucose was notably decreased following circ-ACACA-knockdown relative to the shRNA-NC group. As expected, the expression levels of glycolysis-related proteins, including HIF-1A, LDHA, GLUT1 and HK2, were markedly downregulated following the knockdown of circ-ACACA compared with those in the shRNA-NC group (Fig. 3E). These 
A
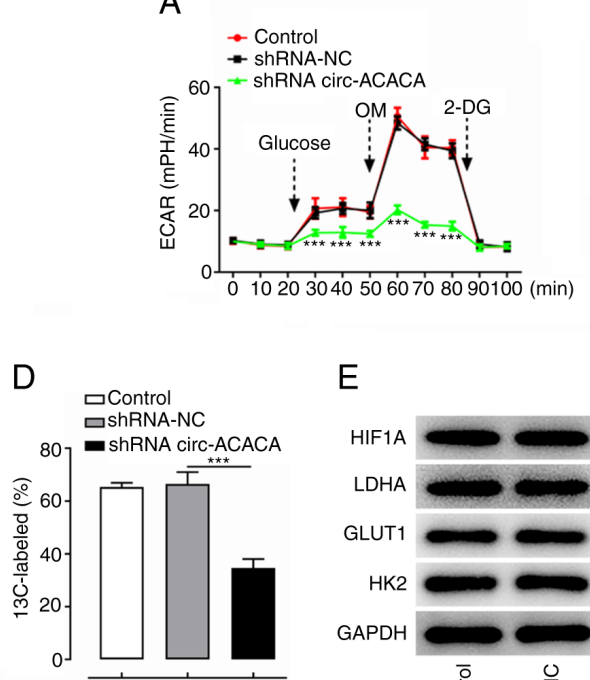
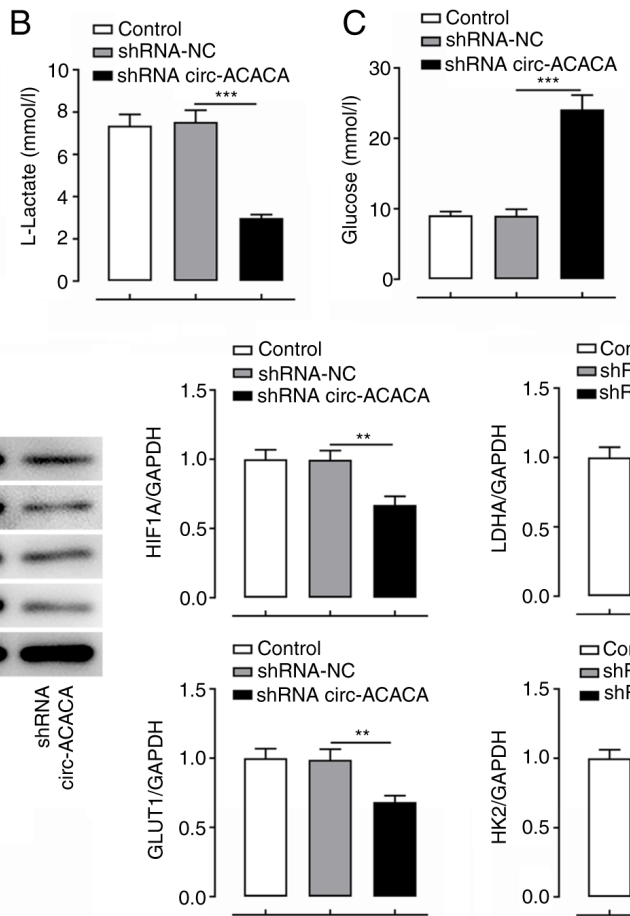

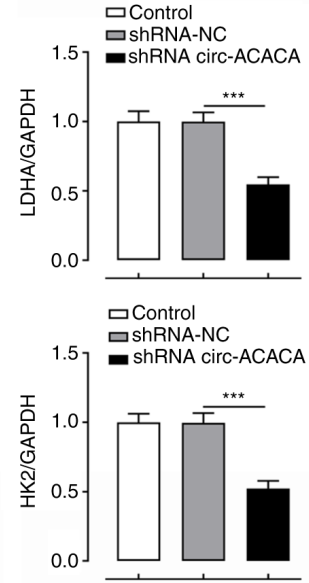

Figure 3. Silencing of circ-ACACA suppresses glycolysis in cervical cancer cells. (A) ECAR after HeLa cells were transfected with shRNA-circ-ACACA. ${ }^{* * *} \mathrm{P}<0.001$ vs. shRNA-NC. (B) Lactate levels and (C) glucose content after HeLa cells were transfected with shRNA-circ-ACACA. (D) Metabolite analysis and (E) glycolysis-related protein expression after HeLa cells were transfected with shRNA-circ-ACACA. ${ }^{* *} \mathrm{P}<0.01,{ }^{* * * *} \mathrm{P}<0.001$. circ-ACACA, circular RNA acetyl-CoA-carboxylase $\alpha$; ECAR, extracellular acidification rate; GLUT1, glucose transporter type 1; HIF1A, hypoxia-inducible factor 1 $\alpha$; HK2, hexokinase-2; LDHA, lactate dehydrogenase A; NC, negative control; shRNA, short hairpin RNA; OM, oligomycin; 2-DG, 2-deoxy-D-glucose.
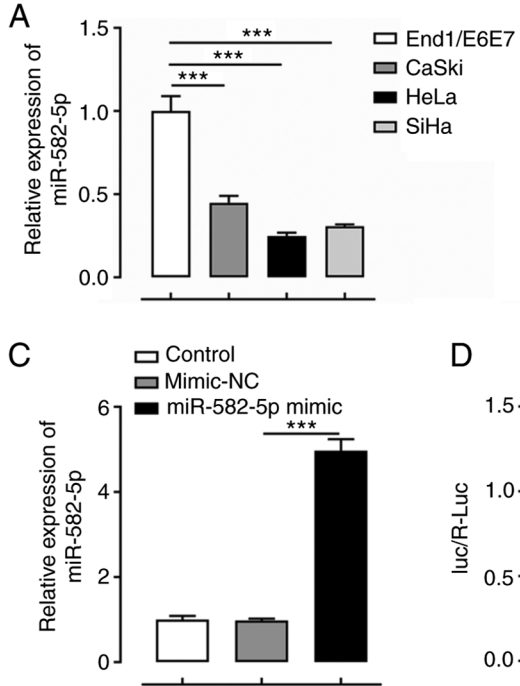

D
B

$\begin{array}{cccc}\text { WT-circ-ACACA: } & 5^{\prime} \text { uagAGCUGCCUUUUACAACUGUAa } & 3^{\prime} \\ & & |:||||||||||| \mid & \\ \text { hsa-miR-582-5p: } & 3^{\prime} & \text { ucaUUGAC--CAACUUGUUGACAUu } & 5^{\prime} \\ \text { MUT-circ-ACACA: } & 5^{\prime} & \text { uagAGCUGCCAAUUUGUUGACAUa } & 3^{\prime}\end{array}$

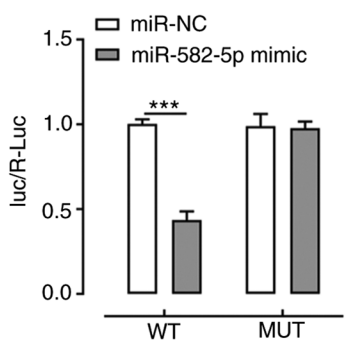

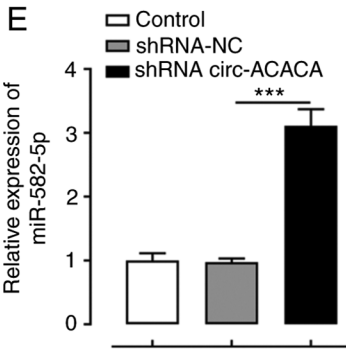

Figure 4. circ-ACACA negatively regulates miR-582-5p expression. (A) Expression levels of miR-582-5p in various cervical cancer cell lines were detected by RT-qPCR. (B) Binding site between circ-ACACA and miR-582-5p predicted by The Encyclopedia of RNA Interactomes database. (C) Expression levels of miR-582-5p were evaluated by RT-qPCR after miR-582-5p mimic was transfected into HeLa cells. (D) Binding between circ-ACACA and miR-582-5p was demonstrated using a dual-luciferase reporter assay. (E) Expression levels of miR-582-5p were examined by RT-qPCR after silencing of circ-ACACA in HeLa cells. ${ }^{* * *} \mathrm{P}<0.001$. circ-ACACA, circular RNA acetyl-CoA-carboxylase $\alpha$; luc/R-Luc, luciferase/Renilla luciferase; miR, microRNA; MUT, mutant; NC, negative control; RT-qPCR, reverse transcription-quantitative PCR; shRNA, short hairpin RNA; WT, wild-type.

results indicated that silencing of circ-ACACA may inhibit glycolysis in CC cells.

circ-ACACA negatively regulates the expression levels of $m i R-582-5 p$. To determine the regulatory effect of circ-ACACA on the expression levels of miR-582-5p, the expression levels of miR-582-5p were first analyzed in various $\mathrm{CC}$ cell lines. The results revealed that miR-582-5p expression was downregulated in CC cells compared with End1/E6E7 cells (Fig. 4A). Subsequently, a binding site between circ-ACACA and miR-582-5p was predicted using ENCORI (Fig. 4B). Following the overexpression of 

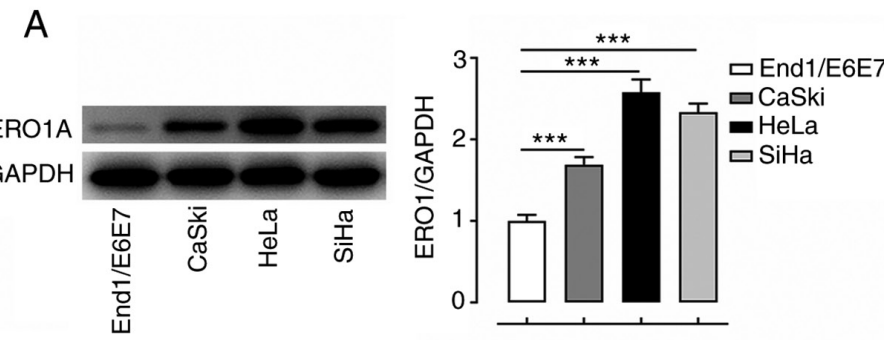

C

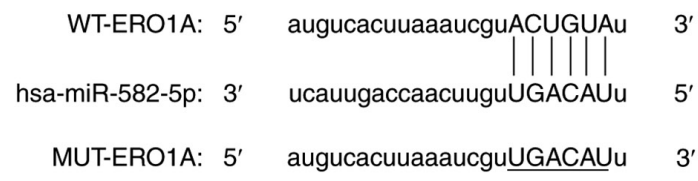

E

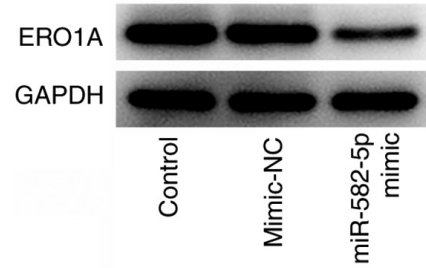

G

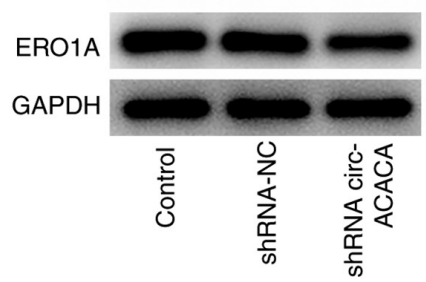

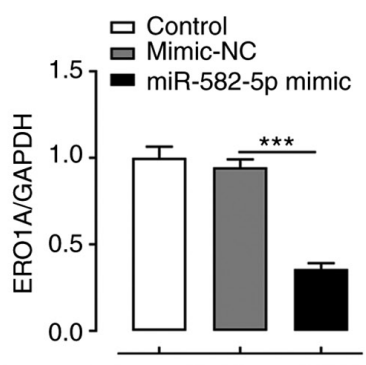

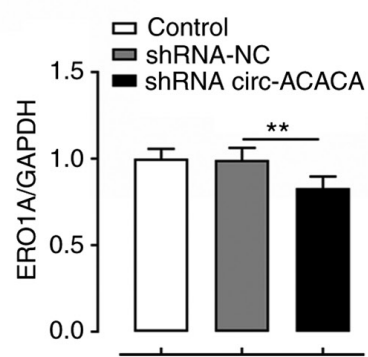

B
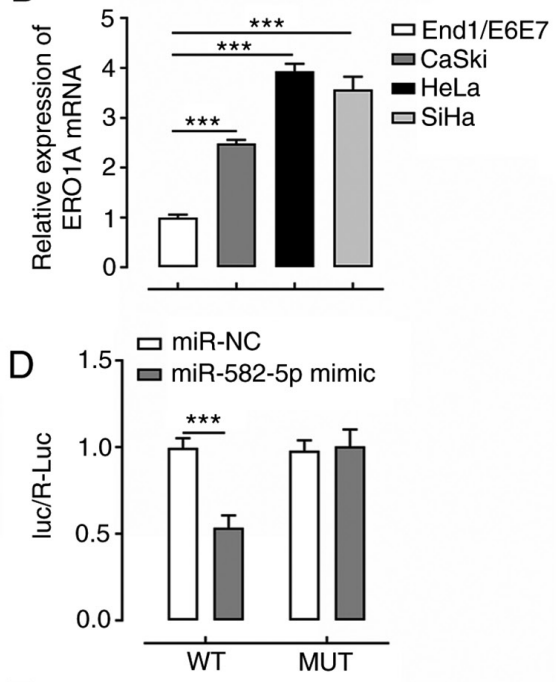

F
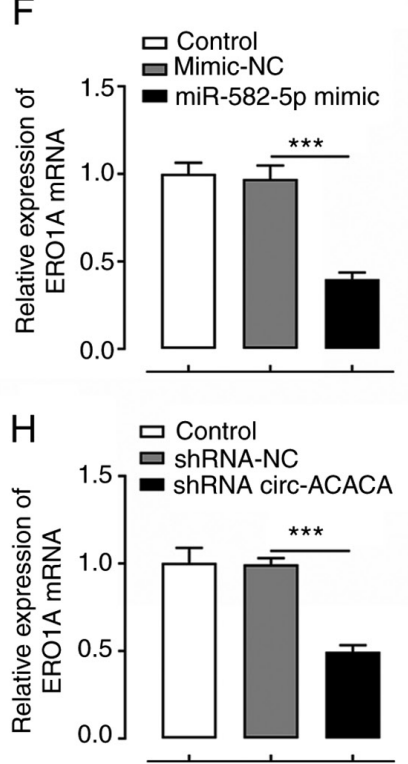

Figure 5.ERO1A is a target of miR-582-5p. (A) Expression levels of ERO1A in various cervical cancer cell lines were assessed by western blotting. (B) Expression of ERO1A in various cervical cancer cell lines was tested with reverse transcription-quantitative PCR. (C) Binding site between miR-582-5p and ERO1A predicted by The Encyclopedia of RNA Interactomes database. (D) Binding between miR-582-5p and ERO1A was demonstrated using a dual-luciferase reporter assay. (E) Expression levels of ERO1A after miR-582-5p mimic transfection into HeLa cells was measured with western blot analysis. (F) ERO1A expression after miR-582-5p mimic transfection into HeLa cells was measured with transcription-quantitative PCR. (G and H) Expression levels of ERO1A after shRNA-circ-ACACA was transfected into HeLa cells. ${ }^{* *} \mathrm{P}<0.01,{ }^{* * *} \mathrm{P}<0.001$. circ-ACACA, circular RNA acetyl-CoA-carboxylase $\alpha$; ERO1A, endoplasmic reticulum disulphide oxidase $1 \alpha$; luc/R-Luc, luciferase/Renilla luciferase; miR, microRNA; MUT, mutant; NC, negative control; shRNA, short hairpin RNA; WT, wild-type.

miR-582-5p by transfection with the miR-582-5p mimic, a dual-luciferase reporter assay was used to demonstrate the binding relationship between circ-ACACA and miR-582-5p (Fig. 4C and D). As shown in Fig. 4E, knockdown of circ-ACACA markedly upregulated the expression levels of miR-582-5p compared with those in the shRNA-NC group. These data suggested that circ-ACACA may negatively regulate miR-582-5p expression in HeLa cells.

ERO1A is a target gene of miR-582-5p. ERO1AIt was hypothesized that circ-ACACA may exert its promoting effects on the progression of $\mathrm{CC}$ via the miR-582-5p/ERO1A signaling axis. To verify this hypothesis, the expression levels of ERO1A in various $\mathrm{CC}$ cell lines were determined. As illustrated in Fig. 5A and B, ERO1A expression was notably unregulated in CC cells compared with End1/E6E7 cells. Since ERO1A was predicted to target and bind with miR-582-5p using ENCORI database. A binding association between ERO1A and miR-582-5p was predicted using ENCORI (Fig. 5C) and verified using a dual-luciferase reporter assay (Fig. 5D). Subsequently, RT-qPCR and western blotting demonstrated that transfection with the miR-582-5p mimic markedly reduced the mRNA and protein expression levels of ERO1A (Fig. 5E and F). Furthermore, transfection with shRNA-circ-ACACA markedly downregulated the mRNA and protein expression levels of ERO1A compared with the shRNA-NC group (Fig. 5G and H). These findings suggested that ERO1A may be a target of miR-582-5p, and that circ-ACACA may upregulate the expression levels of ERO1A by sponging and negatively regulating miR-582-5p expression. 
A
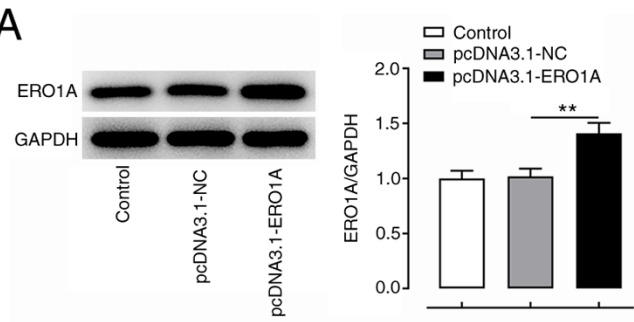

$E$

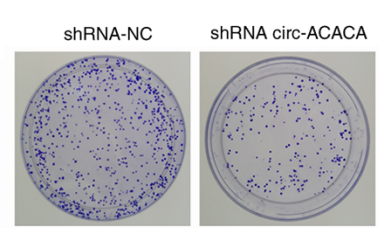

$\mathrm{F}$
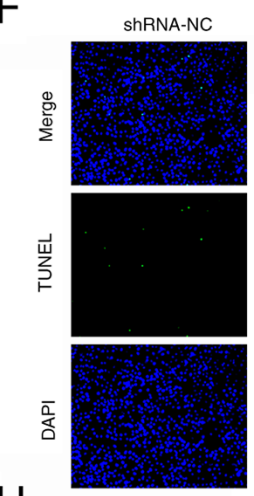

$H$
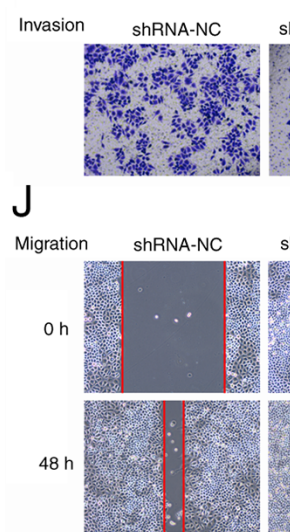

shRNA circ-ACACA
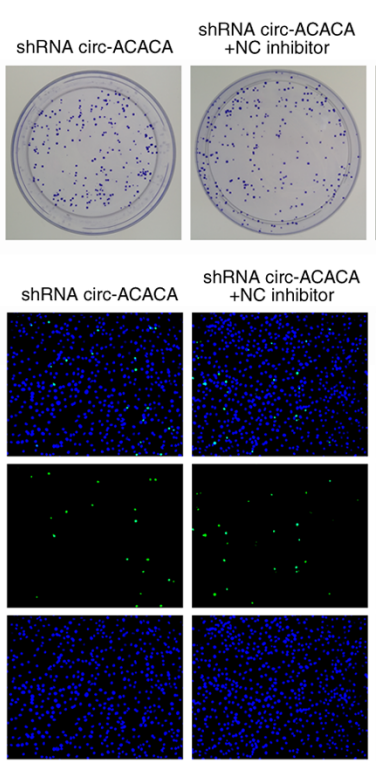

ShRNA circ-ACACA
+NC inhibitor
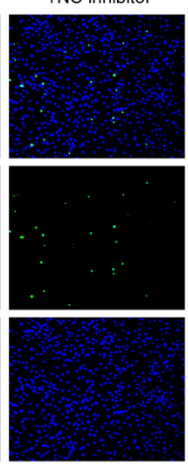

ShRNA circ-ACACA

$+\mathrm{NC}$ inhibitor

ShRNA circ-ACACA
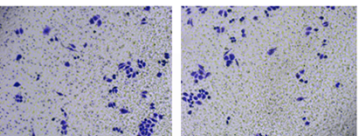

ShRNA circ-ACACA

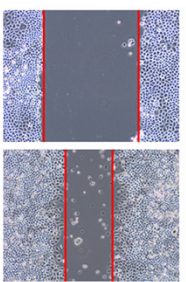

ShRNA circ-ACACA
+ NC inhibitor

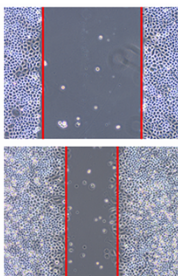

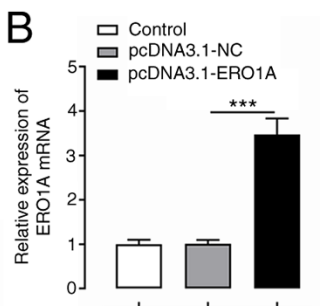

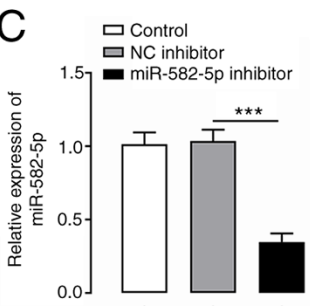

shRNA circ-ACACA ShRNA circ-ACACA

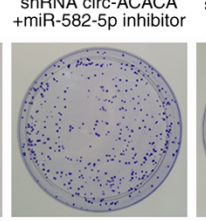

+PCDNA3.1-NC

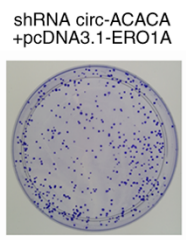

ShRNA circ-ACACA
+ miR-582-5p inhibito
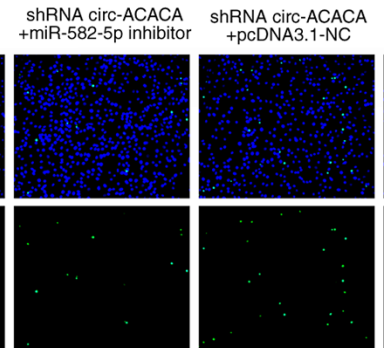

ShRNA circ-ACACA
+pCDNA3.1-ERO1A

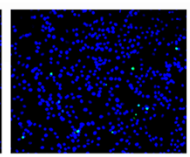

G
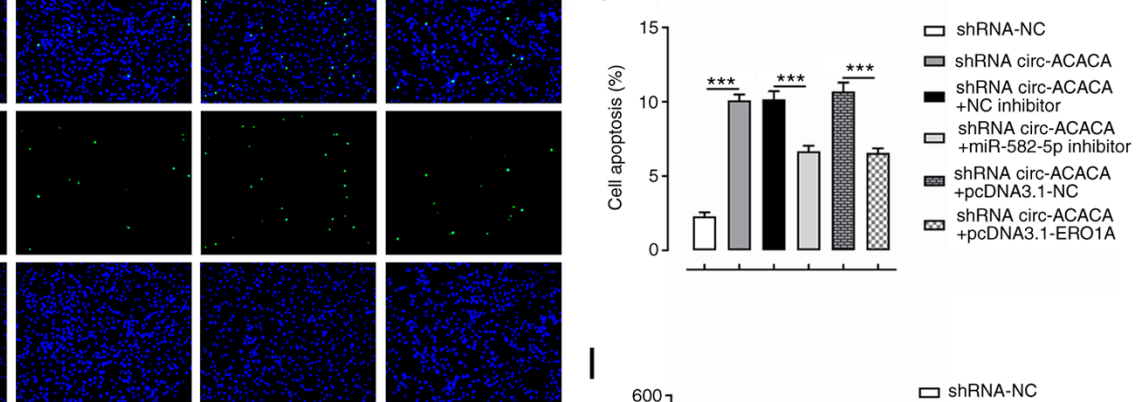

ShRNA circ-ACACA
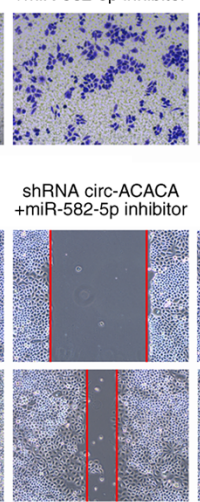
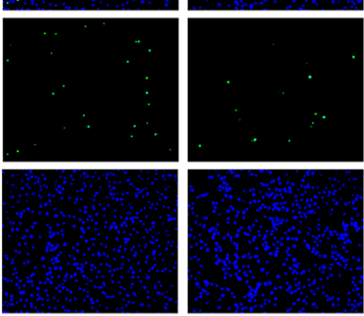

ShRNA circ-ACACA
+PCDNA3.1-NC

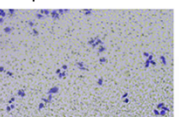

ShRNA CirC-ACACA
+ PCDNA3.-1-ROD1A
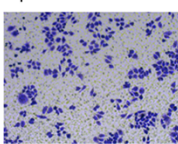

hRNA circ-ACACA
+pCDNA3.1-NC

ShRNA circ-ACACA
+PCDNA3.1-ERO1A
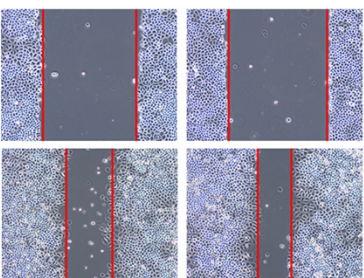

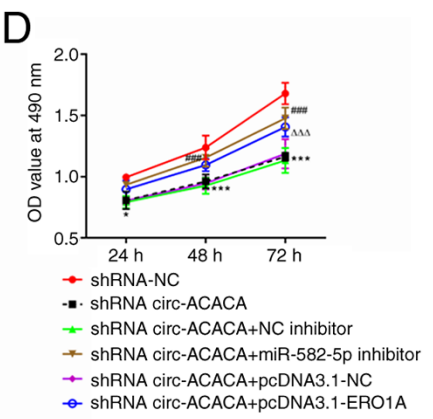

Figure 6. circ-ACACA/miR-582-5p/ERO1A promotes the proliferation, invasion and migration, and inhibits the apoptosis of cervical cancer cells. Expression levels of ERO1A were examined by (A) western blotting and (B) RT-qPCR after pcDNA3.1-ERO1A was transfected into HeLa cells. (C) miR-582-5p expression was evaluated by RT-qPCR after transfection with miR-582-5p inhibitor. ${ }^{* *} \mathrm{P}<0.01,{ }^{* * *} \mathrm{P}<0.001$. (D) Cell viability was detected using an $\mathrm{MTT}$ assay. ${ }^{*} \mathrm{P}<0.05$,

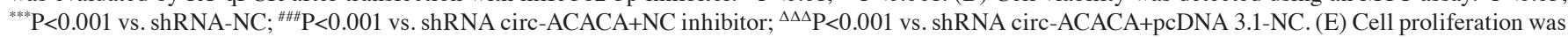
determined using a colony formation assay. (F) Cell apoptosis was evaluated using TUNEL staining. Magnification, x200. (G) The apoptosis rate of TUNEL assay. (H) Cell invasion was evaluated by Transwell assay. (I) The number of invaded cells. Magnification, x100. (J) Cell migration was assessed by wound healing assay. (K) The cell migration rate. Magnification, x100. ${ }^{* * *} \mathrm{P}<0.001$. circ-ACACA, circular RNA acetyl-CoA-carboxylase $\alpha$; ERO1A, endoplasmic reticulum disulphide oxidase $1 \alpha$; miR, microRNA; NC, negative control; OD, optical density; RT-qPCR, reverse transcription-quantitative PCR; shRNA, short hairpin RNA.

Knockdown of miR-582-5p or overexpression of EROIA reverses the effects of circ-ACACA silencing on CC cell proliferation, viability, invasion, migration and apoptosis. To investigate whether circ-ACACA could affect the functions of $\mathrm{CC}$ cells in vitro via the miR-582-5p/ERO1A signaling axis, ERO1A was overexpressed in CC cells. The expression levels of ERO1A were markedly upregulated in the pcDNA3.1-ERO1A group compared with the pcDNA3.1-NC group, demonstrating the successful establishment of
ERO1A-overexpressing CC cells (Fig. 6A and B). Additionally, miR-582-5p expression was notably downregulated after transfection with miR-582-5p inhibitor compared with that in the NC inhibitor group (Fig. 6C). Knockdown of circ-ACACA reduced the viability, proliferation, invasion and migration, and enhanced the apoptosis of HeLa cells, and these effects were all partially reversed following the transfection with the miR-582-5p inhibitor or pcDNA3.1-ERO1A (Fig. 6D-K). In addition, transfection with circ-ACACA reduced the levels of 
A

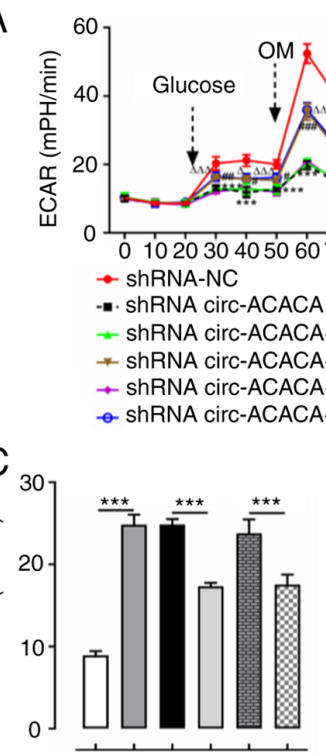

E

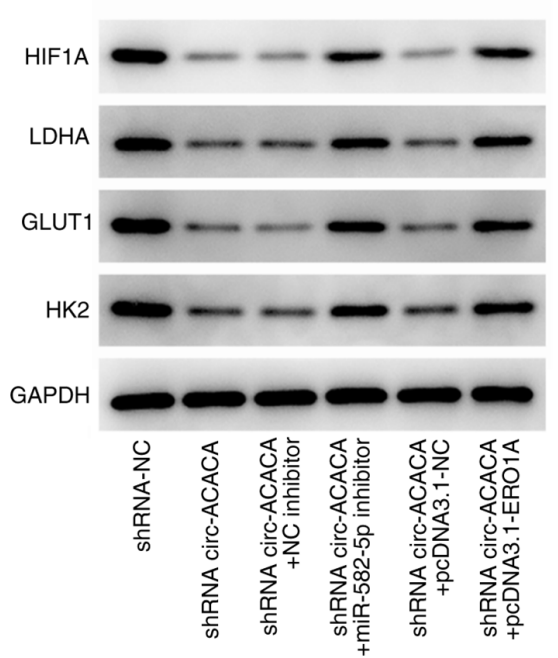

B

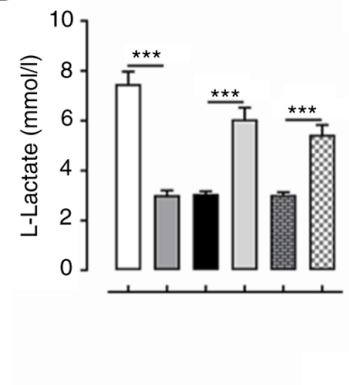

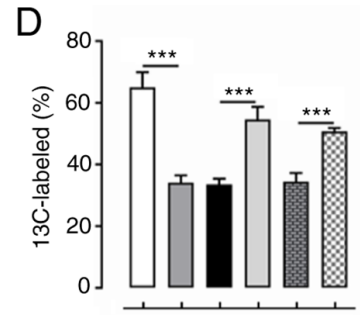

$\square$ ShRNA-NC

$\square$ shRNA circ-ACACA

- shRNA circ-ACACA

+NC inhibitor

ShRNA circ-ACACA

+miR-582-5p inhibitor

shRNA circ-ACACA

+pcDNA3.1-NC

$\omega \begin{aligned} & \text { ShRNA circ-ACACA } \\ & + \text { pcDNA3.1-ERO1A }\end{aligned}$
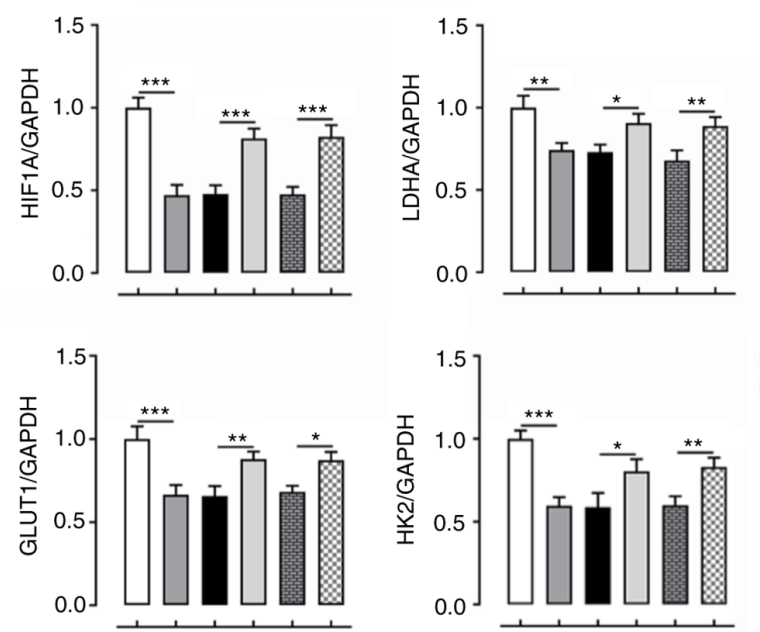

口 ShRNA-NC

$\square$ shRNA circ-ACACA

shRNA circ-ACACA

$+\mathrm{NC}$ inhibitor

ShRNA circ-ACACA

+miR-582-5p inhibito

ShRNA circ-ACACA

+ PCDNA3.1-NC

ShRNA circ-ACACA
+ pCDNA3.1-ERO1A

Figure 7. circ-ACACA/miR-582-5p/ERO1A promotes the glycolysis of cervical cancer cells. (A) ECAR after HeLa cells were transfected with shRNA-circ-ACACA and miR-582-5p inhibitor or pcDNA3.1-ERO1A. ${ }^{*} \mathrm{P}<0.05,{ }^{* *} \mathrm{P}<0.01,{ }^{* * * *} \mathrm{P}<0.001$ vs. shRNA-NC; ${ }^{\# P}<0.05$, ${ }^{\# \#} \mathrm{P}<0.01,{ }^{\# \# "} \mathrm{P}<0.001$ vs. shRNA circ-ACACA+NC inhibitor; ${ }^{\Delta} \mathrm{P}<0.05$ and ${ }^{\Delta \Delta \Delta} \mathrm{P}<0.001$ vs. shRNA circ-ACACA+pcDNA3.1-NC. (B) Lactate levels and (C) glucose content were assessed with commercially available kits. (D) Metabolite analysis and (E) glycolysis-related protein expression after HeLa cells were transfected with shRNA-circ-ACACA and miR-582-5p inhibitor or pcDNA3.1-ERO1A. ${ }^{*} \mathrm{P}<0.05,{ }^{*} \mathrm{P}<0.01,{ }^{* * * *} \mathrm{P}<0.001$. circ-ACACA, circular RNA acetyl-CoA-carboxylase $\alpha$; ECAR, extracellular acidification rate; ERO1A, endoplasmic reticulum disulphide oxidase $1 \alpha$; GLUT1, glucose transporter type 1; HIF1A, hypoxia-inducible factor 1 $\alpha$; HK2, hexokinase-2; LDHA, lactate dehydrogenase A; miR, microRNA; NC, negative control; shRNA, short hairpin RNA; OM, oligomycin; 2-DG, 2-deoxy-D-glucose.

ECAR, and these effects were subsequently reversed by transfection with the miR-582-5p inhibitor or pcDNA3.1-ERO1A (Fig. 7A). Furthermore, the results of the lactate production and glucose consumption assays revealed that the decreased lactate production and enhanced glucose levels induced by transfection with shRNA-circ-ACACA were partially recovered following the transfection with the miR-582-5p inhibitor or pcDNA3.1-ERO1A (Fig. 7B and C). Finally, the shRNA-circ-ACACA-induced decreases in the ratio of ${ }^{13} \mathrm{C}$-marked glucose and expression levels of glycolysis-related proteins were partially reversed following transfection with the miR-582-5p inhibitor or pcDNA3.1-ERO1A (Fig. 7D and E). Overall, these results suggested that knockdown of miR-582-5p or overexpression of ERO1A alleviates the effects of circ-ACACA silencing on CC cell proliferation, viability, invasion, migration and apoptosis.

\section{Discussion}

ncRNAs have been suggested to serve important roles in the progression of numerous types of tumor, such as prostate, non-small cell lung, colorectal and cervical cancer (25-29). circRNAs, which are single-stranded ncRNAs, have been revealed to be involved in the development of multiple cancer types (30). Due to the increased stability, evolutionary conservation and high abundance of circRNAs, they have been established as essential players in numerous physiological and pathophysiological processes (31). Previous research has demonstrated that circ-ACACA expression is upregulated in NSCLC tissues and cells, and silencing of circ-ACACA hinders the cellular functions of NSCLC cells, including proliferation, invasion and migration, via regulation of the miR-1183 and PI3K/AKT signaling pathway (9). Therefore, 
it was hypothesized that circ-ACACA may also serve as an oncogene to promote the tumorigenesis of $\mathrm{CC}$. The results of the present study revealed that circ-ACACA expression was significantly upregulated in CC cells, and circ-ACACA was expressed at a higher level in the cytoplasm compared with the nucleus. Silencing of circ-ACACA inhibited the viability, proliferation, invasion and migration, and enhanced the apoptosis of CC cells.

Alteration of energy metabolism, especially abnormal activation of the glycolysis pathway has been observed in diverse human cancer types, including CC (32-36). Cancer cells are dependent on the glycolytic pathway to meet their high energy demands; thus, increased glycolysis is a hallmark of cancer cells (37). Notably, knockdown of circ-ACACA could hinder proliferation and migration of NSCLC cells and also reduce the glycolysis rate (9). In the present study, the effect of circ-ACACA on glycolysis in CC cells was determined. Higher lactate levels have been previously identified to increase the risk of recurrence and metastasis in patients with CC, and are associated with poor overall survival (38). Furthermore, high lactate content in the tumor microenvironment has been revealed to induce highly acidic conditions, which hinders the function of several chemotherapeutic drugs, such as tamoxifen, cisplatin and adriamycin $(39,40)$. In the present study, silencing of circ-ACACA reduced the ECAR and lactate levels. Increased glucose uptake is another characteristic of cancer cells (37), and the present study revealed that glucose uptake was decreased in HeLa cells transfected with shRNA-circ-ACACA. HIF-1A, LDHA, GLUT1 and HK2 serve important roles in glycolytic metabolism (39). The present study revealed that transfection with shRNA-circ-ACACA downregulated the expression levels of these factors, indicating that shRNA-circ-ACACA may inhibit glycolysis. Therefore, these results indicated the suppressive effect of silencing of circ-ACACA on glycolysis and the progression of $\mathrm{CC}$.

The unique cellular stability and ability of circRNAs to sponge miRNAs and proteins suggests their potential as novel molecular markers for targeted therapies for cancer (40). miRNAs are known to serve an important role in the pathogenesis of most types of cancer (41). Efforts have been made to determine the effect of miR-582 on cancer development (42). A previous study reported that miR-582-5p is differentially expressed during the development of multiple types of cancer, indicating that the expression levels of miR-582-5p depend on the specific type of cancer tissue or cells (42). The results of the present study demonstrated that the expression levels of miR-582-5p were downregulated in $\mathrm{CC}$ cells, and dual-luciferase reporter assays verified the binding between circ-ACACA and miR-582-5p. RT-qPCR analysis revealed that the silencing of circ-ACACA markedly upregulated the expression levels of miR-582-5p, demonstrating the negative association between circ-ACACA and miR-582-5p.

circRNAs have been reported to act as miRNA sponges in circRNA/miRNA/mRNA signaling axes (43). In the present study, bioinformatics analysis was used to predict that ERO1A could interact with miR-582-5p, which prompted further investigations to determine the role of ERO1A in CC cells. ERO1A, which is a protein involved in the oxidative protein folding of molecules involved in cancer progression, has been reported to be induced by hypoxia in HeLa cells (44). The expression levels of ERO1A have been reported to be upregulated in numerous types of cancer cells compared with normal cells, such as breast, pancreatic and colorectal cancer $(19,17,22)$, which was consistent with the present findings that $\mathrm{CC}$ cells exhibited upregulated ERO1A expression compared with End1/E6E7 cells. Notably, transfection with the miR-582-5p mimic downregulated the expression levels of ERO1A in HeLa cells. Whether circ-ACACA affected the functions of HeLa cells via regulation of the miR-582-5p/ERO1A signalling axis was subsequently investigated. The results demonstrated that transfection with shRNA-circ-ACACA decreased the proliferation, invasion and migration, and promoted the apoptosis of HeLa cells, while these effects were reversed following transfection with the miR-582-5p inhibitor or pcDNA3.1-ERO1A.

In conclusion, to the best of our knowledge, the present study was the first to investigate the role of circ-ACACA in $\mathrm{CC}$ development and provided evidence to suggest that the knockdown of circ-ACACA may decrease proliferation, invasion, migration and glycolysis, and promote apoptosis in HeLa cells via targeting of the miR-582-5p/ERO1A signaling axis. These results highlight the potential of circ-ACACA as a novel biomarker for CC. However, the lack of experiments regarding the clinical value of circ-ACACA/miR-582-5p/ERO1A in CC tissue samples to determine its clinical applicability, as well as in vivo antitumor effects in an animal model are limitations of the present study. Additionally, based on a previous study in NSCLC (9), the circ-ACACA/miR-1183/PI3K/protein kinase B signaling pathway and whether there is a direct interaction between circ-ACACA and miR-1183 should also be explored in CC in future studies. Therefore, comprehensive analysis is required in the future.

\section{Acknowledgements}

Not applicable.

\section{Funding}

No funding was received.

\section{Availability of data and materials}

All data generated or analyzed during this study are included in this published article.

\section{Authors' contributions}

DH and CL interpreted the data and performed experiments. DH collected the data, searched the literature, designed the study and wrote the manuscript. CL revised the manuscript. Both authors read and approval the final manuscript. DH and CL confirm the authenticity of all the raw data.

\section{Ethics approval and consent to participate}

Not applicable. 


\section{Patient consent for publication}

Not applicable.

\section{Competing interests}

The authors declare that they have no competing interests.

\section{References}

1. Siegel RL, Miller KD and Jemal A: Cancer statistics, 2019. CA Cancer J Clin 69: 7-34, 2019

2. Torre LA, Bray F, Siegel RL, Ferlay J, Lortet-Tieulent J and Jemal A: Global cancer statistics, 2012. CA Cancer J Clin 65 87-108, 2015.

3. Fan Y, Sheng W, Meng Y, Cao Y and Li R: LncRNA PTENP1 inhibits cervical cancer progression by suppressing miR-106b. Artif Cells Nanomed Biotechnol 48: 393-407, 2020.

4. Fader AN: Surgery in cervical cancer. N Engl J Med 379: 1955-1957, 2018

5. Fan Y, Meng Y, Yang S, Wang L, Zhi W, Lazare C, Cao C and Wu P: Screening of cervical cancer with self-collected cervical samples and next-generation sequencing. Dis Markers 2018 4826547, 2018

6. Wilusz JE and Sharp PA: Molecular biology. A circuitous route to noncoding RNA. Science 340: 440-441, 2013.

7. Jia YJ, Liu MJ and Wang SX: CircRNA hsa_circRNA_0001776 inhibits proliferation and promotes apoptosis in endometria cancer via downregulating LRIG2 by sponging miR-182. Cancer Cell Int 20: 412, 2020

8. Zhang W, Liu T, Li TS and Zhao XD: Hsa_circRNA_102002 facilitates metastasis of papillary thyroid cancer through regulating miR-488-3p/HAS2 axis. Cancer Gene Ther 28: 279-293, 2021.

9. Wu W, Xi W, Li H, Yang M and Yao X: Circular RNA circ-ACACA regulates proliferation, migration and glycolysis in non-small-cell lung carcinoma via miR-1183 and PI3K/PKB pathway. Int J Mol Med 45: 1814-1824, 2020

10. Liu J, Liu S, Deng X, Rao J, Huang K, Xu G and Wang X: MicroRNA-582-5p suppresses non-small cell lung cancer cells growth and invasion via downregulating NOTCH1. PLoS One 14: e0217652, 2019.

11. Huang S, Zou C, Tang Y, Wa Q, Peng X, Chen X, Yang C, Ren D, Huang Y, Liao Z, et al: miR-582-3p and miR-582-5p suppress prostate cancer metastasis to bone by repressing TGF- $\beta$ signaling. Mol Ther Nucleic Acids 16: 91-104, 2019.

12. Wu J, Li W, Ning J, Yu W, Rao T and Cheng F: Long noncoding RNA UCA1 targets miR-582-5p and contributes to the progression and drug resistance of bladder cancer cells through ATG7-mediated autophagy inhibition. Onco Targets Ther 12 495-508, 2019

13. Li L and Ma L: Upregulation of miR-582-5p regulates cell proliferation and apoptosis by targeting AKT3 in human endometrial carcinoma. Saudi J Biol Sci 25: 965-970, 2018.

14. Zhang Y, Huang W, Ran Y, Xiong Y, Zhong Z, Fan X, Wang Z and Ye Q: miR-582-5p inhibits proliferation of hepatocellular carcinoma by targeting CDK1 and AKT3. Tumour Biol 36: 8309-8316, 2015.

15. Tanaka T, Kutomi G, Kajiwara T, Kukita K, Kochin V, Kanaseki T, Tsukahara T, Hirohashi Y, Torigoe T, Okamoto Y, et al: Cancer-associated oxidoreductase ERO1- $\alpha$ promotes immune escape through up-regulation of PD-L1 in human breast cancer. Oncotarget 8: 24706-24718, 2017.

16. Zhang J, Yang J, Lin C, Liu W, Huo Y, Yang M, Jiang SH, Sun Y and Hua R: Endoplasmic reticulum stress-dependent expression of ERO1L promotes aerobic glycolysis in pancreatic cancer. Theranostics 10: 8400-8414, 2020.

17. Han F, Xu Q, Zhao J, Xiong P and Liu J: ERO1L promotes pancreatic cancer cell progression through activating the Wnt/catenin pathway. J Cell Biochem 119: 8996-9005, 2018.

18. Yan W, Wang X, Liu T, Chen L, Han L, Xu J, Jin G, Harada K, Lin $\mathrm{Z}$ and Ren $\mathrm{X}$ : Expression of endoplasmic reticulum oxidoreductase 1- $\alpha$ in cholangiocarcinoma tissues and its effects on the proliferation and migration of cholangiocarcinoma cells. Cancer Manag Res 11: 6727-6739, 2019.
19. Zilli F, Marques Ramos P, Auf der Maur P, Jehanno C, Sethi A, Coissieux MM, Eichlisberger T, Sauteur L, Rouchon A, Bonapace L, et al: The NFIB-ERO1A axis promotes breast cancer metastatic colonization of disseminated tumour cells. EMBO Mol Med 13: e13162,2021.

20. Zhang Y, Li T, Zhang L, Shangguan F, Shi G, Wu X, Cui Y, Wang X, Wang X, Liu Y, et al: Targeting the functional interplay between endoplasmic reticulum oxidoreductin-1 $\alpha$ and protein disulfide isomerase suppresses the progression of cervical cancer. EBioMedicine 41: 408-419, 2019.

21. Seol SY, Kim C, Lim JY, Yoon SO, Hong SW, Kim JW, Choi SH and Cho JY: Overexpression of Endoplasmic Reticulum Oxidoreductin 1- $\alpha$ (ERO1L) is associated with poor prognosis of gastric cancer. Cancer Res Treat 48: 1196-1209, 2016.

22. Takei N, Yoneda A, Sakai-Sawada K, Kosaka M, Minomi K and Tamura Y: Hypoxia-inducible ERO1 $\alpha$ promotes cancer progression through modulation of integrin- $\beta 1$ modification and signalling in HCT116 colorectal cancer cells. Sci Rep 7: 9389, 2017.

23. Livak KJ and Schmittgen TD: Analysis of relative gene expression data using real-time quantitative PCR and the 2(-Delta Delta C(T)) method. Methods 25: 402-408, 2001.

24. Yamamoto T, Takano N, Ishiwata K, Ohmura M, Nagahata Y, Matsuura T, Kamata A, Sakamoto K, Nakanishi T, Kubo A, et al: Reduced methylation of PFKFB3 in cancer cells shunts glucose towards the pentose phosphate pathway. Nat Commun 5: 3480, 2014.

25. Guarnerio J, Zhang Y, Cheloni G, Panella R, Mae Katon J, Simpson M, Matsumoto A, Papa A, Loretelli C, Petri A, et al: Intragenic antagonistic roles of protein and circRNA in tumorigenesis. Cell Res 29: 628-640, 2019.

26. Zhang G, Liu Y, Yang J, Wang H and Xing Z: Inhibition of circ_0081234 reduces prostate cancer tumor growth and metastasis via miR-1/MAP3K1 axis. J Gene Med: e3376, 2021.

27. Yang C, Shi J, Wang J, Hao D, An J and Jiang J: Circ 0006988 promotes the proliferation, metastasis and angiogenesis of non-small cell lung cancer cells by modulating miR-491-5p/MAP3K3 axis. Cell Cycle 20: 1334-1346, 2021.

28. Wang J, Li S, Zhang G and Han H: Sevoflurane inhibits malignant progression of colorectal cancer via hsa circ 0000231-mediated miR-622. J Biol Res (Thessalon) 28: 14, $202 \overline{1}$.

29. Xie J,Chen Q,Zhou PandFanW:CircularRNA hsa_circ_0000511 improves epithelial mesenchymal transition of cervical cancer by regulating hsa-mir-296-5p/HMGA1. J Immunol Res 2021: 9964538, 2021.

30. Ding L, Zhao Y, Dang S, Wang Y, Li X, Yu X, Li Z, Wei J, Liu M and Li G: Circular RNA circ-DONSON facilitates gastric cancer growth and invasion via NURF complex dependent activation of transcription factor SOX4. Mol Cancer 18: 45, 2019.

31. Li Y, Zheng F, Xiao X, Xie F, Tao D, Huang C, Liu D, Wang M, Wang L, Zeng F and Jiang G: CircHIPK3 sponges miR-558 to suppress heparanase expression in bladder cancer cells. EMBO Rep 18: 1646-1659, 2017.

32. Pelicano H, Martin DS, Xu RH and Huang P: Glycolysis inhibition for anticancer treatment. Oncogene 25: 4633-4646, 2006.

33. Hua Q, Mi BM, Xu F, Wen J, Zhao L, Liu J and Huang G: Hypoxia-induced lncRNA-AC020978 promotes proliferation and glycolytic metabolism of non-small cell lung cancer by regulating PKM2/HIF-1 $\alpha$ axis. Theranostics 10: 4762-4778, 2020.

34. Sun SF, Li WW, Ma XM and Luan H: Long noncoding RNA LINC00265 promotes glycolysis and lactate production of colorectal cancer through regulating of miR-216b-5p/TRIM44 axis. Digestion 101: 391-400, 2020.

35. Shang RZ, Wang M, Dai B, Du J, Wang J, Liu Z, Qu S, Yang X, Liu J, Xia C, et al: Long noncoding RNA SLC2A1-AS1 regulates aerobic glycolysis and progression in hepatocellular carcinoma via inhibiting the STAT3/FOXM1/GLUT1 pathway. Mol Oncol 14: 1381-1396, 2020.

36. Xu J, Tan QQ and Lie T: USP 22 promotes the expression of GLUT1 and HK2 to facilitate growth and glycolysis in cervical cancer cells. Eur J Gynaecol Oncol 41: 790-796, 2020.

37. Yeung C, Gibson AE, Issaq SH, Oshima N, Baumgart JT, Edessa LD, Rai G, Urban DJ, Johnson MS, Benavides GA, et al: Targeting glycolysis through inhibition of lactate dehydrogenase impairs tumor growth in preclinical models of ewing sarcoma. Cancer Res 79: 5060-5073, 2019.

38. Walenta S, Wetterling M, Lehrke M, Schwickert G, Sundfør K, Rofstad EK and Mueller-Klieser W: High lactate levels predict likelihood of metastases, tumor recurrence, and restricted patient survival in human cervical cancers. Cancer Res 60: 916-921, 2000. 
39. Wang M, Wang W, Wang $\mathrm{J}$ and Zhang $\mathrm{J}$ : MiR-182 promotes glucose metabolism by upregulating hypoxia-inducible factor $1 \alpha$ in NSCLC cells. Biochem Biophys Res Commun 504: 400-405, 2018.

40. Kristensen LS, Hansen TB, Veno MT and Kjems J: Circular RNAs in cancer: Opportunities and challenges in the field. Oncogene 37: 555-565, 2018.

41. Rupaimoole R, Calin GA, Lopez-Berestein G and Sood AK: miRNA deregulation in cancer cells and the tumor microenvironment. Cancer Discov 6: 235-246, 2016.

42. Tian Y, Guan Y, Su Y, Luo W, Yang G and Zhang Y: MiR-582-5p inhibits bladder cancer-genesis by suppressing TTK Expression. Cancer Manag Res 12: 11933-11944, 2020.
43. Tang W, Ji M, He G, Yang L, Niu Z, Jian M, Wei Y, Ren L and $\mathrm{Xu}$ J: Silencing CDR1as inhibits colorectal cancer progression through regulating microRNA-7. Onco Targets Ther 10: 2045-2056, 2017.

44. Takei N, Yoneda A, Kosaka M, Sakai-Sawada K and Tamura Y: ERO1 $\alpha$ is a novel endogenous marker of hypoxia in human cancer cell lines. BMC Cancer 19: 510, 2019.

(i) (3) This work is licensed under a Creative Commons Attribution-NonCommercial-NoDerivatives 4.0 International (CC BY-NC-ND 4.0) License. 